\title{
Hybrid metal nanostructure arrays for colour printing
}

\author{
K. Wilson, C. A. Marocico, E. Pedreuza, C. Smith and A. L. Bradley \\ School of Physics, Trinity College Dublin, College Green, Dublin 2, Ireland \\ Tel: (00 353) 1896 2881,e-mail: kewilson@tcd.ie
}

\begin{abstract}
Nanoscale metasurfaces have become an area of growing interest in recent years with the promise of new functionalities. One area that has received a lot of attention is sub-diffraction limit printing. We demonstrate the fabrication of a novel hybrid Au-Ag metal nanoparticle array design on an ultra-thin film propylene polymer material. The design presented generates strong colours due to the plasmonic response and coupling between the two metallic components. It is sensitive to the polymer layer thickness, and it is shown that by tuning this layer thickness, the colour response of the nanostructure can be carefully engineered. We determine the effect fabrication has on the optical properties of the polymer material at these ultra-thin thicknesses, $<200 \mathrm{~nm}$. The fabrication of violet, cyan and red colour pixels using an $\mathrm{Au}$ nanoparticle array and $\mathrm{Ag}$ backreflector is demonstrated. We utilise colour conversion software to convert spectra of fabricated samples to RGB values on the CIE 1931 colour space and values agree with observed colour from optical microscope images.
\end{abstract}

Keywords: Hybrid Nanostructure, Plasmonic Array, Localised Surface Plasmon Resonance, Colour generation

\section{INTRODUCTION}

It is well known that metal nanoparticles with subwavelength dimensions show strong localised surface plasmon resonance features in the electromagnetic spectrum. These plasmonic resonances can be tuned across the visible domain by altering their shape and size and by controlling the surrounding refractive index. In more recent times nanoscale lithographic techniques, allow for the fabrication of high fidelity arrays of the metallic nanoparticles [1]. These arrays of nanoparticles, often hole-disk coupled systems, form plasmonic pixels and colour images with sub-diffraction limit printing resolutions have been demonstrated [2-4]. Arrays also provides additional parameters which can be tuned to adjust the reflected colours, such as the pitch. This can potentially be exploited for a variety of applications, such as low power consumption displays for smart-phones and other handheld devices, consequently extending their battery lifetime, as well as sensing and anti-counterfeit measures.

While the principle of colour generation from plasmonic pixels is not new, the route to realistic implementation has been unclear. A wide range of metal nanostructure shapes and sizes can be considered, though some can introduce unwanted polarization effects [5] or angular effects [6,7], which can reduce the viewing angle over which the colour is reproducible and clear. The achievable colour range is also a key criterion for good colour rendering. This is a limiting factor in many current plasmonic colour printing methods, particularly those based on using a single metal.

By utilising a structure with nanoparticles of sub-wavelength dimensions and pitch, the viewing angle of colours produced is improved. The possibility to use a hybrid metal system is also interesting, for example one which can benefit from strong Au plasmonic resonance response and the high reflectivity of Ag across the visible spectrum. Such a structure will be explored in this paper, as the novel design utilises the benefits of a two metal structure separated by a dielectric material, a design that has not been demonstrated previously. Au is resistant to tarnishing, and does not suffer from photobleaching. The high Ag reflectivity also helps to recycle light back toward the gold nanodisk to generate a stronger plasmonic response. These material advantages allow for the creation of a plasmonic nanostructure with strong plasmonic interaction in the visible domain that is robust and has the potential for varied device integration in many fields.

Structures have been designed based on Au nanodisks separated by an ultra-thin polymer layer from a continuous Ag film. The polymer layer also prevent tarnishing of the Ag layer. ${ }^{[8]}$ The reflectivity of the structure is highly sensitive to the thickness of the polymer layer that separates the metals, and by tuning this thickness the colour response of the nanostructure can be engineered. The layer is typically less than $400 \mathrm{~nm}$. Though hybrid plasmonic metal-metal [9-11] systems have been examined in detail, they are often employed as connected or alloyed materials, rather than having controlled separation of metallic components for colour generation as in this work. Therefore, the practical realisation of the plasmonic pixel requires the considerably challenging fabrication of an array of Au nanodisks on an ultrathin propylene polymer layer, as opposed to other examples which use hard dielectrics such as $\mathrm{SiO}_{2}$ thin film layers [12,13]. In this paper the fabrication and characterisation of the hybrid metallic structures is presented, demonstrating colour tunability across the visible range.

\section{Fabrication}

A schematic of a complete structure is shown in Fig. 1. The substrate is a plane (100) CZ grown Silicon wafer, sonicated in solutions of Acetone, Methanol and Isopropyl Alcohol (IPA), respectively, for 20 minutes each before being dried with a Nitrogen gun to ensure no residue on the surface. 
After the deposition of a 100nm Ag film, a propylene based polymer layer was deposited. This layer was subsequently covered with a spin-coated layer of PMMA of $250 \mathrm{~nm}$, followed by 2 minutes at $180^{\circ} \mathrm{C}$ on a hotplate. The PMMA is used as a positive tone resist to generate nanostructure array patterns. E-Spacer was used to prevent excess charge build up, to improve the resolution of finished arrays. The nanodisk array patterns were then completed using e-beam lithography (EBL). The patterns consisted of disks of $175 \mathrm{~nm}$ diameter, with a pitch of $300 \mathrm{~nm}$.

After exposure, E-Spacer was removed with a rinse in de-ionised water for 10 seconds. The pattern was developed using a 1:3 solution of Methyl Isobutyl Ketone (MIBK) to IPA for 45 seconds. The development process was stopped by submerging the sample in IPA, followed by drying with a Nitrogen gun. Subsequently, a $100 \mathrm{~nm}$ thick Au layer was deposited, before lift-off was undertaken to remove the remaining PMMA and unwanted $\mathrm{Au}$ from the substrate. All three presented samples were fabricated using this method, with three different polymer thicknesses used.

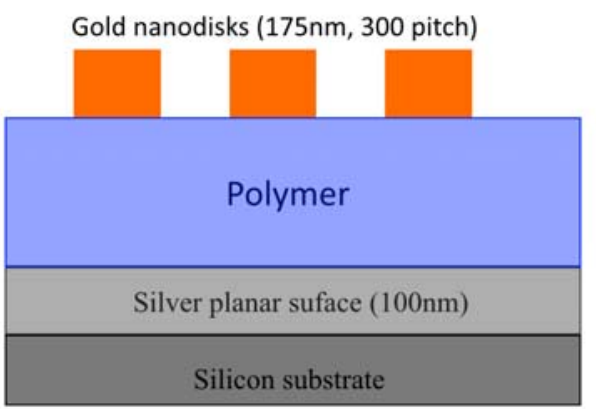

Figure 1. Side view schematic of hybrid nanostructure.

\section{Discussion}

\subsection{Geometry characterisation}

The structural properties of the samples were characterised. Multiple angle scan microspot ellipsometry was carried out to determine the polymer thickness in each of the fabricated structures. The lateral spot size was 300 $\mu \mathrm{m}$. The measurements returned values of $(70 \pm 5) \mathrm{nm},(138 \pm 5) \mathrm{nm}$ and $(196 \pm 5) \mathrm{nm}$ for the thickness of the polymer layer in an area near the patterned array on the substrate.

EBL fabrication on such a thin polymer film is challenging, and it is important to determine if the fabrication process itself modifies the properties of the polymer layer. In particular, if the structure, and consequently, optical properties of the polymer layer are altered by exposure to high energy electrons during the EBL process, or if changes are induced chemically by the development or lift-off procedures. As measurements could not be taken of the area just directly below the Au nanodisks, a test sample was prepared consisting of a Si substrate, $100 \mathrm{~nm}$ Ag layer, $300 \mathrm{~nm}$ polymer layer and PMMA layer. A $3 \times 3 \mathrm{~mm}^{2}$ area of the sample was then exposed using EBL, followed by the same chemical treatment used for the patterned samples. The test sample was then analysed using the ellipsometry techniques. The thickness of polymer layer was measured on the area of exposure, as well as at a position near the exposed area. The thickness of the polymer on the exposed area was found to be $(292 \pm 5) \mathrm{nm}$, which corresponds well to that of $(288 \pm 5) \mathrm{nm}$ measured on a close by area that was not exposed. This indicates there is no significant thickness change undergone by the polymer due to the EBL patterning. The refractive index of the material was also measured at these two positions, and in this case the measurements reduction of the real refractive index of approximately0.1 had occurred in the visible range, shown in Fig.2 (a).

There was an increase in imaginary component of the refractive index on the exposed area of polymer, particularly at shorter wavelengths of the visible spectrum, as can be seen in the inset of Fig. 2 (a). The spectral profile of the $\mathrm{k}$ values indicates scattering rather than absorption in a material, as the values are significantly higher in the UV region than at longer wavelengths. This suggests that the surface roughness is increased as a result of the EBL process, though the scattering remains relatively low in both cases, with the exposed area averaging an extinction $(\mathrm{k})$ across the measured range of 0.0471 compared to 0.0271 for the unexposed area.

Finally, the structures fabricated were imaged using scanning electron microscopy. A micrograph of sample $\mathrm{C}$ is shown in Fig. 2(b). As can be seen nanodisks have been successfully fabricated on these thin polymer layers of thickness less than $200 \mathrm{~nm}$. The fabricated nanodisks are found to have good definition and a diameter of 175 $\mathrm{nm}$. The nanodisks are arranged in a square array with $300 \mathrm{~nm}$ pitch. 

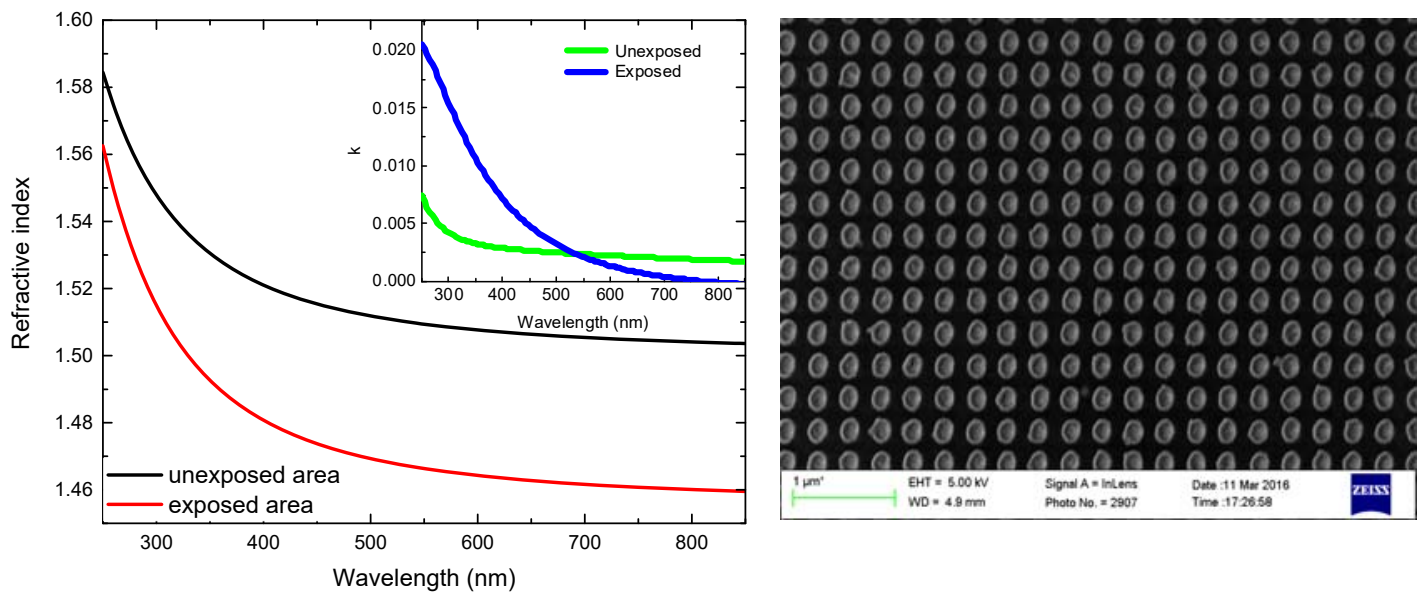

Figure 2.(a) Refractive index for exposed and unexposed areas of polymer, measured using ellipsometry (Inset: imaginary $k$ component of refractive index for each area). (b) SEM image of a fabricated array, consisting of a silicon substrate, 100nm Ag layer, 196nm polymer layer thickness and Au nanodisks with height of 100nm and diameter of $175 \mathrm{~nm}$

\subsection{Optical characterisation}

Having successfully fabricated the structures, the arrays were optically characterised. Optical microscope images for each of the three samples are presented in Fig. 3. Distinct violet, blue and red colours are observed for polymer layer thicknesses of $70 \mathrm{~nm}$ (Sample A), $138 \mathrm{~nm}$ (Sample B) and $196 \mathrm{~nm}$ (Sample C), respectively. As can be seen, the colours are more or less uniform across the areas.

The corresponding reflectance spectra were measured using a Xenon lamp and are presented in Fig. 4. Samples $\mathrm{A}$ and $\mathrm{B}$ show higher reflectance in the blue part of the visible spectrum and lower reflectance in the red than Sample C. However, the exact shade and colour that will be observed in reflection cannot be easily inferred from the reflectance spectrum, as the incident light source will not have equal intensity across the spectrum and the human eye is not equally sensitive across the spectrum. Therefore, taking account of these parameters the reflectance spectra have been converted to colours on the CIE 1931 RGB colourspace map.

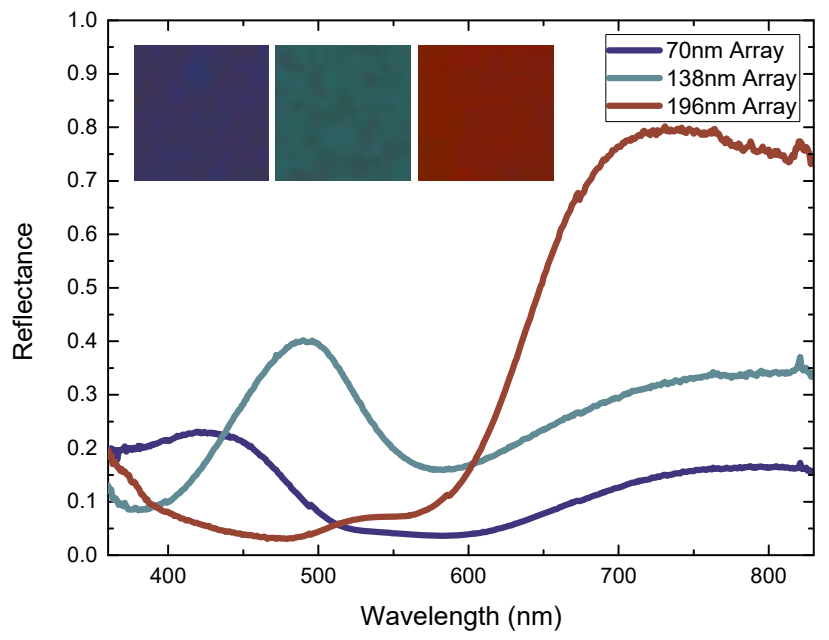

Figure 3. Spectra of arrays measured by reflectometry, with line colour representing colour response of each array by colour conversion software. (Insets: $10 \mu \mathrm{m}$ sections of Samples A, B and C imaged using optical microscope. Polymer layers of $70 \mathrm{~nm}$ (left) $138 \mathrm{~nm}$ (middle) and 196nm (right) shown.)

Integrating functions, that act as colour matching functions, separately corresponding to the "Red", "Green" and "Blue" (RGB) colour sensing cone cells present in the human eye are used to find the relative intensity of a given spectrum for each colour component. These integrating functions match with sensitivity functions of the CIE 1931 RGB standard colourspace map, a standard for the perceived colour in human colour vision. The 
spectrum is integrated by each function, and the value returned by each is used to map on to the CIE colourspace map in a reproducible result that is representative of the colour experienced by an observer.

RGB colourspace values found were $(64,53,126)$ for Sample A, $(96,139,149)$ for Sample B and $(151,68,51)$ for Sample C. The line colour in Fig. 3 represents these calculated values as they determined from the reflectance spectra. It can be noted that these colours are in close agreement with the optical microscope images.

\section{CONCLUSIONS}

We have presented a method for the fabrication of metallic nanodisk arrays directly onto ultrathin propylene based polymer layers by electron beam lithography. This allows for a facile method of fabrication of hybrid metallic nanostructure, in which the two different metals are separated by less than $200 \mathrm{~nm}$. Using structures based on a Ag back reflector and Au nanodisks, reflected colour was generated across the visible spectrum. It was shown that for a fixed nanodisk diameter the colours could be tuned by changing only the polymer thickness, with violet, cyan and red demonstrated.

\section{ACKNOWLEDGEMENTS}

Mitsui Chemicals Group for supplying propylene polymer material used. This work was supported by Science Foundation Ireland (SFI) under grant numbers 13/TIDA/12717 and 10/IN.1/12975. KW acknowledges a postgraduate research scholarship from the Irish Research Council (GOIPG/2013/851).

\section{REFERENCES}

[1] J. Martin, J. Plain: Fabrication of Aluminium nanostructure for plasmonics, J. Phys. D: Appl. Phys. 48, 184002,2015

[2] B. Lu, et al:: High-resolution plasmonic structural colors from nanohole arrays with bottom metal disks, Optics Letters, Vol 41, No 7, 2016

[3] K. Kumar, et al:: Printing colour at the optical diffraction limit, Nature Technology, pp. 557-561, 2012

[4] S. J. Tan, et al.: Plasmonic color palettes for photorealistic printing with aluminum nanostructures, Nano Letters, 14, 4023-4029, 2014.

[5] Y. Gu, et al.: Color generation via subwavelength plasmonic nanostructures, Nanoscale, 7, pp. 6409-6419, 2015

[6] Y. R. Wu, et al.: Angle-insensitive structural colours based on metallic nanocavities and coloured pixels beyond the diffraction limit, Scientific Reports, 3, 1194, 2013

[7] J. Clausen, et al.: Plasmonic Metasurfaces for Coloration of Plastic Consumer Products, Nano Letters, 14 , 4499-4504, 2014

[8] M.D. McMahon, et al.: Rapid tarnishing of silver nanoparticles in ambient laboratory air, Applied Physics $B$, pp. 915-921, 2005.

[9] S. Cha, et al:: Au-Ag Core-Shell Nanoparticle Array by Block Copolymer Lithography for Synergistic Broadband Plasmonic Properties, ACS Nano, 9 (5), pp 5536-5543, 2015

[10] A. Kuzma, et al.: Plasmonic properties of Au-Ag nanoparticles: distinctiveness of metal arrangements by optical study, J. Appl. Phys. 115, 053517, 2014

[11] L. Sun, et al.: Surface plasmon enhanced quantum transport in a hybrid metal nanoparticle array, Physics Letters A 378 2708-2712, 2014

[12] B. Zhang, et al.: Polarization-independent dual-band infrared perfect absorber based on a metal-dielectricmetal elliptical nanodisk array, Optics Express, Vol 19, No. 16, 2011

[13] Y. Huang, et al.: Aluminum Plasmonic Multicolor Meta-Hologram, Nano Letters, 15, pp 3122-3127, 2015 